\title{
Bonding nature of metal/oxide incoherent interfaces by first- principles calculations
}

$\operatorname{AUTHOR}(\mathrm{S}):$

Matsunaga, K; Sasaki, T; Shibata, N; Mizoguchi, T; Yamamoto, T; Ikuhara, Y

\section{CITATION:}

Matsunaga, K ...[et al]. Bonding nature of metal/oxide incoherent interfaces by first-principles calculations. PHYSICAL REVIEW B 2006, 74(12): 125423.

\section{ISSUE DATE:}

2006-09

URL:

http://hdl.handle.net/2433/39888

RIGHT:

Copyright 2006 American Physical Society 
PHYSICAL REVIEW B 74, 125423 (2006)

\title{
Bonding nature of metal/oxide incoherent interfaces by first-principles calculations
}

\author{
Katsuyuki Matsunaga, ${ }^{1, *}$ Takeo Sasaki, ${ }^{2}$ Naoya Shibata, ${ }^{3}$ Teruyasu Mizoguchi, ${ }^{3}$ Takahisa Yamamoto, ${ }^{4}$ and Yuichi Ikuhara ${ }^{3}$ \\ ${ }^{1}$ Department of Materials Science and Engineering, Kyoto University, Yoshida-honmachi, Sakyo-ku, Kyoto 606-8501, Japan \\ ${ }^{2}$ Department of Materials Engineering, The University of Tokyo, 2-11-16, Yayoi, Bunkyo-ku, Tokyo 113-8656, Japan \\ ${ }^{3}$ Institute of Engineering Innovation, The University of Tokyo, 2-11-16, Yayoi, Bunkyo-ku, Tokyo 113-8656, Japan \\ ${ }^{4}$ Department of Advanced Materials Science, Graduate School of Frontier Science, The University of Tokyo, 5-1-5, \\ Kashiwanoha, Kashiwa-shi, Chiba 227-8561, Japan \\ (Received 23 May 2006; revised manuscript received 23 July 2006; published 27 September 2006)
}

\begin{abstract}
A bonding mechanism of large-mismatched metal/oxide heterointerfaces, classified as incoherent interfaces, is investigated by first-principles calculations. As a model system, incoherent $\mathrm{Ni} / \mathrm{ZrO}_{2}(111)$ interfaces are selected, and the interfacial bonding characters and their relevance to the interface strength are analyzed. It is found that the chemical bonds of the interfacial atomic pairs are strongly dependent on the atomic configurations in the interface structures, and show a site-dependent character from ionic through covalent/metallic bonding. Thus, even in the presence of a large misfit, stable interfaces can be formed by an effective chemical bonding transition along the interfaces. First-principles tensile tests show that such a bonding multiplicity strongly affects the atomic-scale fracture behavior and ideal mechanical strength of the interfaces.
\end{abstract}

DOI: 10.1103/PhysRevB.74.125423

PACS number(s): 73.20.- r, 73.43.Cd

Heterointerfaces are important components in microstructures of advanced materials such as composites and coatings, and their interfacial properties often determine overall performance and reliability of the materials systems. Among heterointerfaces, combination of metal and metal-oxide has been extensively studied experimentally and theoretically, ${ }^{1,2}$ in order to obtain detailed knowledge on bonding characteristics across the interfaces. However, the interfacial bonding is generally difficult to describe simply by a particular chemical bonding state such as metallic or ionic, because the two constituent materials have dissimilar electronic structures. Moreover, differences in crystal structures and lattice parameters may also affect the interfacial bonding state. Therefore, it is essential to reveal a generic and detailed bonding mechanism of metal/oxide interfaces.

From a theoretical viewpoint, a number of metal/oxide interface systems have been studied by the first-principles method, together with a supercell approach. ${ }^{1-8}$ However, although most of real metal/oxide interfaces contain lattice misfits, previous theoretical calculations dealt with specific coherent interface models without lattice misfits. In this case, several symmetric coherent structures (all metal atoms are located at certain symmetric sites on an oxide surface) were assumed in supercells. As a matter of course, coherent interface models contain a particular type of interfacial atomicpair configuration such as on top, hollow, and bridging, and thus the bonding state over the interface plane can also be understood by its particular character of bonding.

In contrast, real metal/oxide interfaces with lattice misfits undergo misfit strains, and the strains are relaxed in a semicoherent or incoherent manner. In the semicoherent case (typically, several percent of lattice misfit), it is known that a two-dimensional network of misfit dislocations is introduced to accommodate the misfit strain, and the interface areas between misfit dislocations can be regarded as coherent structures. Based on this situation, Hong et al. performed coherent-model calculations of $\mathrm{Ag} / \mathrm{MgO}(100)$ interfaces, which is semicoherent in reality, and the calculated proper- ties were corrected by the energy of misfit dislocations. ${ }^{3}$ More recently, Benedek et al. calculated the semicoherent $\mathrm{Cu} / \mathrm{MgO}\{222\}$ oxygen-terminated interface, and showed that the interface properties can be represented by average of interface properties for different symmetric coherent structures. 9

On the other hand, when a lattice misfit of a metal/oxide interface exceeds $10 \%$ or more, the interface structure cannot be described by arrays of misfit dislocations as found in semicoherent interfaces. ${ }^{10}$ In this case, unlike in coherent or semicoherent interfaces, long-range lattice continuity across the interface is missing, and the interface structure is referred to as incoherent. Originally, a truly incoherent interface implies a complete lack of lattice continuity and bonding across an interface plane, and thus adhesion of metal and oxide cannot be realized. ${ }^{11}$ However, a number of interfaces such as $\mathrm{Cu}(111) / \mathrm{Al}_{2} \mathrm{O}_{3}(0001)$ and $\mathrm{Ni}(111) / \mathrm{YSZ}(111)$ were experimentally found to form incoherent structures. ${ }^{12-15}$ The real incoherent interfaces exhibit specific orientation relationships and adhesion, which is evidence for the presence of particular interfacial bonding states. Due to worse lattice continuity of incoherent interfaces compared to coherent or semicoherent ones, various kinds of metal-cation or metaloxygen bonding pairs in on-top, hollow, and bridging configurations and so forth coexist and play an important role for interface adhesion. In order to understand properties of incoherent metal/oxide interfaces, such characteristic bond configurations across the interface plane should be taken into account.

Bonding and adhesion properties of large-mismatched metal/oxide interfaces were often explained qualitatively by differences in lattice parameters and elastic constants between metal and oxide lattices. ${ }^{2,11,16,17}$ Also, the image charge theory ${ }^{1,2}$ is used to qualitatively understand the interfacial bonding, but the above arguments do not explicitly take account of detailed interfacial atomic configurations and their bonding states. Moreover, as stated above, most of previous first-principles studies on metal/oxide interfaces are 
limited to coherent cases, and thus the physical origin of adhesion in incoherent metal/oxide interfaces is poorly understood so far.

In this study, bonding characteristics and the associated mechanical properties of incoherent metal/oxide interfaces are investigated in a first-principles manner. In interface modeling for theoretical calculations, it is important to use supercells appropriate for incoherent metal/oxide interfaces. For this purpose, the coincidence boundary model is used here, which assumes that, even in an incoherent interface, an interface-energy minimum will occur when a fraction of interfacial atoms in a softer crystal lattice are slightly relaxed and occupy specific coincidence positions on a stiffer crystal surface. ${ }^{18}$ A large-mismatched metal/oxide interface can now be described by a basic unit whose length parallel to the interface plane is $m a_{\mathrm{o}}{ }^{0} \approx n a_{\mathrm{m}}{ }^{0}$, where $m$ and $n$ are integers, and $a_{\mathrm{o}}{ }^{0}$ and $a_{\mathrm{m}}{ }^{0}$ are unit lengths of oxide-lattice and metallattice planes perpendicular to an interface, respectively. A misfit parameter $\delta$ in this case is defined by $\delta=\left(m a_{\mathrm{o}}{ }^{0}\right.$ $\left.-n a_{\mathrm{m}}{ }^{0}\right) / n a_{\mathrm{m}}{ }^{0}$. Therefore, it is necessary to select an incoherent metal/oxide system for which first-principles calculations are feasible and in which the $\delta$ value can be minimized as small as possible, using smaller integers of $m$ and $n$.

Based on the above requirement for supercell modeling, a number of metal/oxide interfaces studied experimentally so far were reviewed, and eventually the interface system of $\mathrm{Ni}(111) / \mathrm{ZrO}_{2}(111)$ is selected as an optimum incoherent model of this study. In our previous report, the experimental high-resolution transmission electron microscopy (HRTEM) image of the $\mathrm{Ni} /$ cubic yttria-stabilized $\mathrm{ZrO}_{2}$ (YSZ) interface showed that this interface having a lattice misfit of $31 \%$ is atomically sharp with a cube-on-cube orientation relationship (OR) of $(111)_{\mathrm{Ni}} \|(111)_{\mathrm{YSZ}}$ and $[1 \overline{1} 0]_{\mathrm{Ni}} \|[1 \overline{1} 0]_{\mathrm{YSZ}}$, and no misfit dislocations are observed along the interface plane, indicating the incoherent structure. ${ }^{15}$ For this incoherent system, a misfit-free condition of $\delta=0$ in the coincidence boundary model cannot be attained even for large $m$ and $n$ values, but a coincidence repeat unit with a rather small misfit of $\delta=-2.7 \%$ can be obtained by using relatively small integers of $m=2$ and $n=3$ along the $\langle 1 \overline{1} 0\rangle$ direction. Accordingly, repeat units of $\mathrm{ZrO}_{2}(111)$ and $\mathrm{Ni}(111)$ parallel to the interface plane are extended by $2 \times 2$ and $3 \times 3$ along $\langle 1 \overline{1} 0\rangle$, respectively, in the supercell construction. Supercell configurations viewed normal to the interface plane are displayed in Fig. 1. The remaining misfit of $\delta=-2.7 \%$ is compensated by compressing the Ni slabs parallel to the interface plane, because the $\mathrm{ZrO}_{2}$ lattice is stiffer than the Ni lattice. It can be said that the original lattice misfit between $\mathrm{Ni}$ and $\mathrm{ZrO}_{2}$ (31\%) can be minimized up to $2.7 \%$ by using the extended interface supercell.

Even for the extended supercell of the interface, there are a number of possible interface configurations with respect to relative translation states between $\mathrm{Ni}$ and $\mathrm{ZrO}_{2}$ lattices. In reality, it is reasonable to describe the incoherent structure by a mixture of different translation states. In the present interface model, the interface configuration can be repeated by a translation vector of $\mathbf{t}=a_{\mathrm{ZrO} 2} / 3[\overline{2} 11]$, and two different translation states of $T_{0}$ and $T_{1}$ are considered as representative (a)

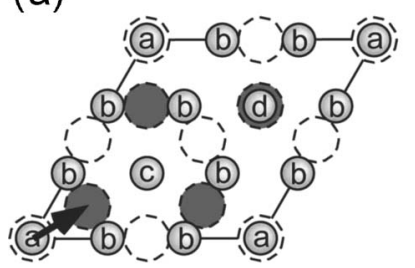

(b)
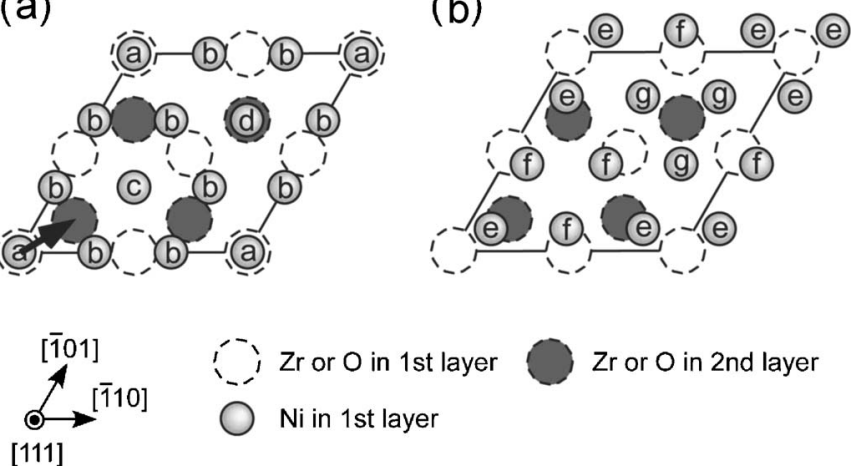

Zr or O in 1st layer

$\mathrm{Zr}$ or $\mathrm{O}$ in 2nd layer

$\mathrm{Ni}$ in 1st layer

FIG. 1. Atomic arrangement of the interface supercells, projected on the interface plane. (a) the translation state of $T_{0}$ and (b) of $T_{1}$. In this figure, one Ni layer and two $\mathrm{ZrO}_{2}$ layers adjacent to the interface are only shown. The arrow in (a) indicates a minimum translation vector of $\mathbf{t}=a_{\mathrm{ZrO} 2} / 3[\overline{2} 11]$. In each model, Ni atoms located at equivalent sites on the $\mathrm{ZrO}_{2}$ surface are assigned by using alphabetical characters from " $a$ " to " $g$."

translated structures, where the $T_{1}$ structure is obtained by a translation of $2 \mathbf{t} / 3$ from $T_{0}$. The atomic positions of $\mathrm{Ni}$ in $T_{0}$ can be represented by four kinds of positions on $\mathrm{ZrO}_{2}$ (111) $[a-d$ in Fig. 1(a)]; an on-top site (a), an asymmetric site slightly off from the on-top location $(b)$ and two kinds of hollow sites $c$ and $d$ (the $d$ site is atop an atom in the second $\mathrm{ZrO}_{2}$ layer). In contrast, the atomic configurations across the interface plane in $T_{1}$ are quite different from those in $T_{0}$, where $\mathrm{Ni}$ atoms are situated at three kinds of asymmetric positions on the $\mathrm{ZrO}_{2}$ surface $[e-g$ in Fig. 1(b)].

Each interface supercell comprises a $\mathrm{ZrO}_{2}(111)$ slab sandwiched by $\mathrm{Ni}(111)$ slabs. Since $\{111\}$ planes of $c-\mathrm{ZrO}_{2}$ are polar planes composed of $\mathrm{Zr}$ or $\mathrm{O}$ atoms, $\mathrm{Zr}$ and $\mathrm{O}$ terminated interfaces are considered in this study. In order to avoid spurious effects of dipole moments on atomic structures and energies of the slab models, the $\mathrm{Zr}$ and $\mathrm{O}$ terminated $\mathrm{ZrO}_{2}$ slabs have identical surface termination, and then contain ten and nine $\mathrm{ZrO}_{2}$ (111) layers, respectively. It is noted that the $\mathrm{O}$ terminated slab is stoichiometric, and the $\mathrm{Zr}$ terminated one is $\mathrm{Zr}$ rich. In order to make identical interface configurations at both ends of the $\mathrm{ZrO}_{2}$ slab, the Ni slab with seven layers is employed for the $T_{0}$ structure, while the one with six layers is employed for $T_{1}$. The resulting total numbers of atoms in the supercells range from 90 to 103, depending on the $\mathrm{ZrO}_{2}$ termination and the translation states. In order to reveal intrinsic and fundamental bonding states at the incoherent interfaces, inclusion of yttrium and oxygen vacancies, which would be important for real Ni/YSZ systems, is not explicitly taken into account in the present study.

Spin-polarized electronic structure calculations of the interface system are performed using the Vienna Ab-Initio Simulation Package (VASP) program. ${ }^{19}$ The projector augmented wave method, ${ }^{20,21}$ which is an all-electron frozencore-type method, is used to represent inner electrons, and electronic wave functions are expanded by plane waves up to a kinetic energy cutoff of $500 \mathrm{eV}$. The generalized gradient approximation (GGA) is used for the exchange-correlation potential, in which the GGA functional proposed by Perdew et al. is used.22 For the hexagonal-shaped interface super- 
cells, Brillouin-zone (BZ) sampling is performed on a 4 $\times 4 \times 1$ Monkhorst-Pack (MP) mesh centered at a $\Gamma$ point (four irreducible $k$ points). ${ }^{23}$ Convergence tests using a 6 $\times 6 \times 1$ MP mesh (seven irreducible $k$ points) for the $\mathrm{O}$ terminated interfaces showed that differences in total energies and stresses of supercells are less than $1.6 \mathrm{meV} / \mathrm{atom}$ and $0.2 \mathrm{GPa}$, respectively. Works of separation $\left(W_{\text {sep }}\right)$ for the interface cleavage, which are a measure of interface strength, are calculated from total-energy differences between the interface supercells and the individual $\mathrm{Ni}$ and $\mathrm{ZrO}_{2}$ surface slabs with their fully relaxed atomic positions. It is found that the $W_{\text {sep }}$ convergence for the denser $k$-point sampling $\left(6 \times 6 \times 1 \mathrm{MP}\right.$ mesh) is less than $0.01 \mathrm{~J} / \mathrm{m}^{2}$. The above results ensure a good accuracy of the present supercell calculations to describe the interface structures and properties.

Excess energies $\gamma$ of the interfaces are evaluated from total energies $\left(E_{\mathrm{T}}\right)$ of interface supercells and atomic chemical potentials $(\mu)$ for constituent atoms as follows.

$$
\gamma=\left(E_{\mathrm{T}}-n_{\mathrm{Zr}} \mu_{\mathrm{Zr}}-n_{\mathrm{O}} \mu_{\mathrm{O}}-n_{\mathrm{Ni}} \mu_{\mathrm{Ni}}\right) / A .
$$

In Eq. (1), $A$ is an interface area, and $n_{i}$ is the number of atoms $i$ in the supercell. As will be shown later, $\mathrm{ZrO}_{2}$ (111) surfaces are also calculated in this study, where a vacuum layer of $1.6 \mathrm{~nm}$ thickness is introduced in the supercell to obtain $E_{\mathrm{T}}$ for the surfaces. The values of $\mu_{\mathrm{Zr}}, \mu_{\mathrm{O}}$, and $\mu_{\mathrm{Ni}}$ are constrained with equilibrium conditions of $\mu_{\mathrm{ZrO}_{2}}=\mu_{\mathrm{Zr}}+2 \mu_{\mathrm{O}}$ and $\mu_{\mathrm{Ni}}=\mu_{\mathrm{Ni} \text { (bulk) }}$, and thus $\mu_{\mathrm{O}}$ ranges from $\mu_{\mathrm{O}}=\mu_{\mathrm{O}_{2} \text { (gas) }} / 2$ (oxidation limit) to $\mu_{\mathrm{O}}=\left(\mu_{\mathrm{ZrO}_{2}}-\mu_{\mathrm{Zr} \text { (bulk) }}\right) / 2$ (reduction limit). $\mu_{\mathrm{O}_{2} \text { (gas) }}$ and $\mu_{\mathrm{Zr} \text { (bulk) }}$ are obtained from total-energy calculations for $\mathrm{O}_{2}$ molecule and hcp $\mathrm{Zr}$ metal.

To begin with, excess energies of $\mathrm{ZrO}_{2}$ (111) surfaces and $\mathrm{Ni} / \mathrm{ZrO}_{2}$ (111) interfaces are evaluated as a function of oxygen chemical potential, as shown in Fig. 2. It can be seen from Fig. 2(a) that the $\mathrm{O}$ terminated surface is most stable over the entire range of $\mu_{\mathrm{O}}$, and the $\mathrm{Zr}$ terminated surface exhibit a fairly high energy even in the reduction limit. This indicates that the clean $\mathrm{ZrO}_{2}$ (111) surface always undergoes $\mathrm{O}$ termination, irrespective of oxygen chemical potentials. When the interface between $\mathrm{ZrO}_{2}$ and $\mathrm{Ni}$ is formed [Fig. 2(b)], the $\mathrm{O}$ terminated structure is also more stable in higher $\mu_{\mathrm{O}}$, whereas the $\mathrm{Zr}$ terminated interface becomes more stable in the reduced condition of $\mu_{\mathrm{O}}<-8.3 \mathrm{eV}$. It is noted that the $T_{0}$ and $T_{1}$ structures for the two termination cases exhibit quite similar interface energies (an energy difference within $0.1 \mathrm{~J} / \mathrm{m}^{2}$ ). It can be said that the interface termination is likely reversed from $\mathrm{O}$ to $\mathrm{Zr}$ termination under the reduced atmosphere, although the $\mathrm{ZrO}_{2}$ (111) surface always has the $\mathrm{O}$ terminated structure. Both of the $\mathrm{Zr}$ and $\mathrm{O}$ terminated interfaces can be formed, depending on oxygen chemical potential. The similar situation was found in the previous theoretical study of the $\mathrm{Nb} / \mathrm{Al}_{2} \mathrm{O}_{3}$ system. ${ }^{6}$

The relaxed structures of the $\mathrm{Zr}$ and $\mathrm{O}$ terminated interfaces are depicted in Fig. 3, together with the interfacial $W_{\text {sep }}$ values. For the $\mathrm{Zr}$ terminated interfaces, $W_{\text {sep }}$ values are around $4.4 \mathrm{~J} / \mathrm{m}^{2}$, irrespective of the translation states, and are about $88 \%$ of $W_{\text {sep }}\left(5.01 \mathrm{~J} / \mathrm{m}^{2}\right)$ for the $\mathrm{Zr}$ terminated nonstoichiometric $\mathrm{Ni} / \mathrm{ZrO}_{2}(100)$ system calculated by Beltrán et al. ${ }^{8}$ The lattice misfit of the present interface $(31 \%)$ is

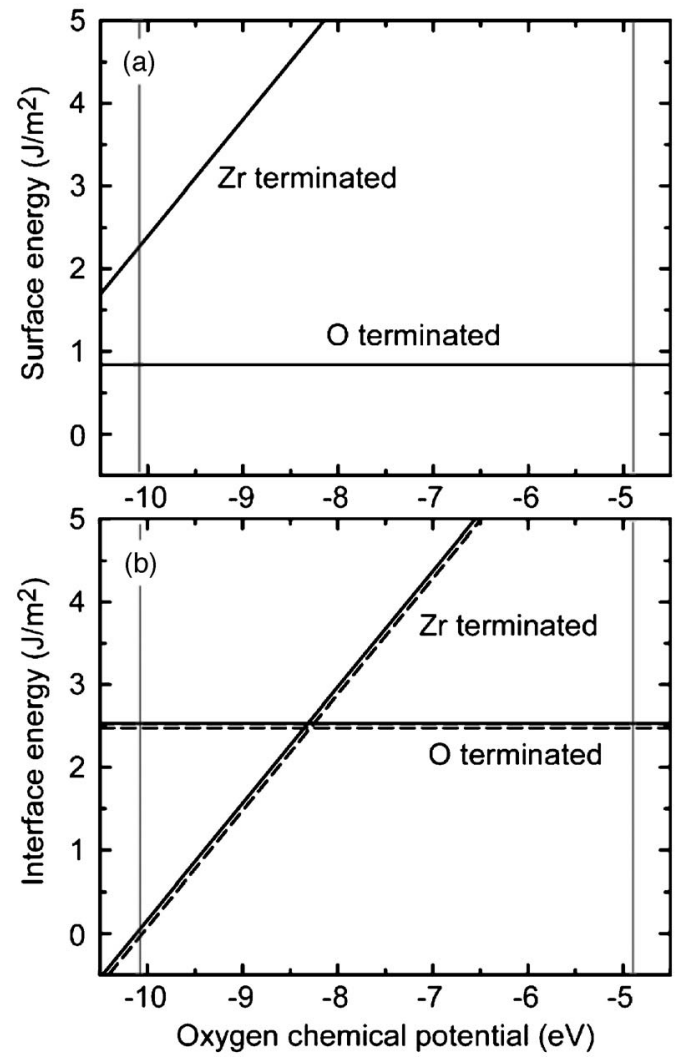

FIG. 2. Excess energies of (a) $\mathrm{ZrO}_{2}$ (111) surfaces and (b) $\mathrm{Ni} / \mathrm{ZrO}_{2}(111)$ interfaces as a function of oxygen chemical potential $\left(\mu_{\mathrm{O}}\right)$. Vertical thin lines indicate $\mu_{\mathrm{O}}$ values at the oxidation and reduction limits. In the plot of (b), solid lines denote energies for the $T_{0}$-type $\mathrm{Zr}$ or $\mathrm{O}$ terminated interfaces, while broken lines indicate results for the $T_{1}$-type interfaces (also see Fig. 1).

much larger than that of the $\mathrm{Ni} / \mathrm{ZrO}_{2}(100)$ interface (less than $2 \%$ ), and yet the $W_{\text {sep }}$ values are not so much reduced as expected from the lattice-misfit difference.

In contrast, the $O$ terminated (111) interfaces exhibit $W_{\text {sep }}$ of about $0.5 \mathrm{~J} / \mathrm{m}^{2}$, which are much smaller than for the $\mathrm{O}$ terminated (100) interface $\left(W_{\text {sep }}=5.74 \mathrm{~J} / \mathrm{m}^{2}\right) .{ }^{8}$ This is mainly due to stoichiometry effects of the interfaces. In general, nonstoichometric interfaces show larger $W_{\text {sep }}$ than stoichiometric ones, due to considerable charge transfer occurring across interfaces. ${ }^{6}$ This is also the case for the present $\mathrm{O}$ terminated interface: the (100) system in Ref. 8 is $\mathrm{O}$ rich while the (111) system here stoichiometric. Therefore, it is not straightforward to make direct comparison of $W_{\text {sep }}$ with Ref. 8, regarding the $\mathrm{O}$ terminated cases.

From Fig. 3, a number of structural characteristics in the incoherent interfaces can be observed. The $\mathrm{Ni}$ and $\mathrm{Zr}$ atoms (O atoms) in the first interface layers of the $\mathrm{Zr}$ terminated $(\mathrm{O}$ terminated) interfaces undergo large relaxations perpendicular to the interface planes. In particular, $\mathrm{Ni}$ atoms tend to rumple more considerably than $\mathrm{Zr}$ or $\mathrm{O}$ atoms, so that the $\mathrm{Ni}$ interface layers are not completely flat. In addition, bond lengths of $\mathrm{Ni}-\mathrm{Zr}$ or $\mathrm{Ni}-\mathrm{O}$ pairs across the interface are dependent on the $\mathrm{Ni}$ sites, which range from $0.24 \mathrm{~nm}$ to $0.30 \mathrm{~nm}$ for the $\mathrm{Zr}$ terminated interfaces, and from $0.19 \mathrm{~nm}$ to $0.30 \mathrm{~nm}$ for the $\mathrm{O}$ terminated ones. These results 

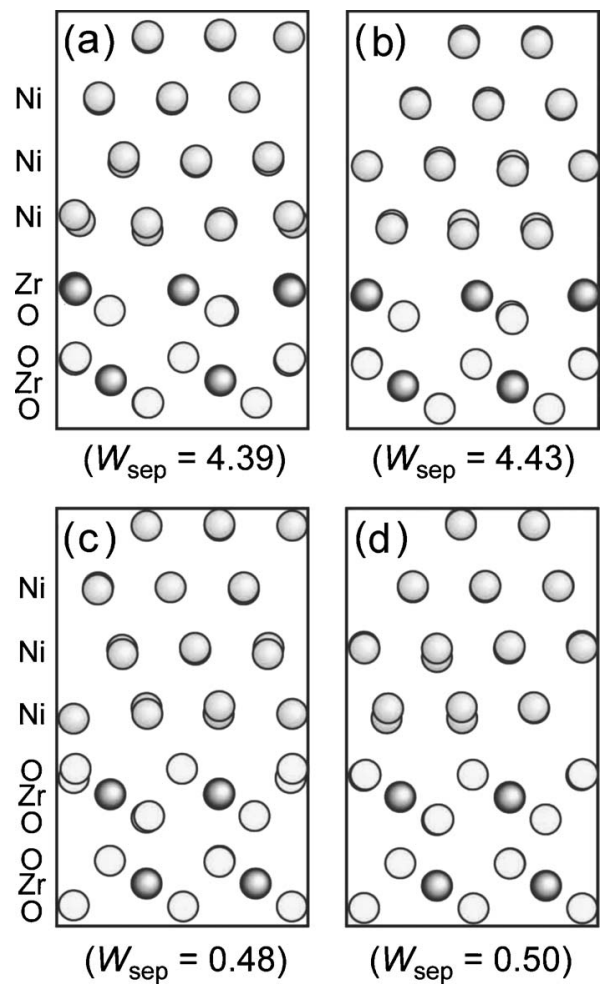

FIG. 3. Relaxed interface structures for $\mathrm{Zr}$ terminated and $\mathrm{O}$ terminated interfaces, viewed along the [101] direction. (a) The $\mathrm{Zr}$ terminated interface in the $T_{0}$ translation state, and (b) the one in $T_{1}$. In the similar way, the results for the $T_{0}$ and $T_{1}$ translation states for the $\mathrm{O}$ terminated case are drawn in (c) and (d), respectively. Works of separation $W_{\text {sep }}$ (unit: $\mathrm{J} / \mathrm{m}^{2}$ ) are shown in the parentheses below the respective structures.

indicate that chemical environments of the $\mathrm{Ni}$ atoms in the interface layers are quite different, depending on the $\mathrm{Ni}$ sites on the $\mathrm{ZrO}_{2}$ (111) surfaces.

In order to reveal electronic structures of the interfaces, atom-projected partial densities of states (PDOSs) are plotted in Fig. 4. For the $\mathrm{Zr}$ and $\mathrm{O}$ terminated interfaces having the $T_{0}$-type structure, PDOS profiles for $\mathrm{Ni}, \mathrm{Zr}$, or $\mathrm{O}$ atoms at the center of each slab (denoted as "bulk"), interfacial $\mathrm{Ni}$ at the on-top site $\left(\mathrm{Ni}^{a}\right)$, interfacial $\mathrm{Zr}$ or $\mathrm{O}$ bonded to $\mathrm{Ni}^{a}$ are only shown. It is worth mentioning here that the PDOS shapes for the interfacial atoms in the two termination cases are different from those for the atoms in bulk, which indicates characteristic atomic interactions at the interfaces. In particular, the Ni-3d band is located around the band gap of $\mathrm{ZrO}_{2}\left(E_{g}\right.$ in Fig. 4), and then the metal-induced gap states (MIGSs) are observed in the PDOS profiles for interfacial $\mathrm{Zr}$ and $\mathrm{O}$. The appearance of MIGSs is closely related to formation of the interfacial bonding.

Similarly to interfacial $\mathrm{Zr}$ or $\mathrm{O}$, the PDOS shapes of the interfacial $\mathrm{Ni}$ atom are also different from that of bulk $\mathrm{Ni}$. In the PDOS of bulk Ni, it can be seen that the majority-spin band (the upper panel) is located lower in energy than the minority-spin one (the lower panel), due to electronexchange interactions. The resultant magnetic moment (MM) of bulk Ni is found to be $0.59 \mu_{\mathrm{B}}$ atom, and is in good agreement with the previous calculations for $\mathrm{Ni}^{24,25}$ However, the

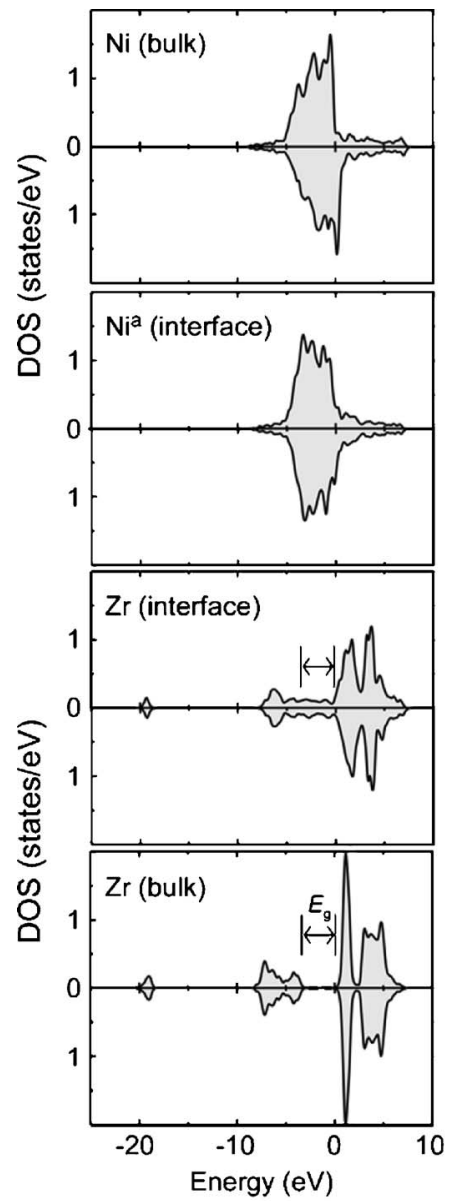

(a)

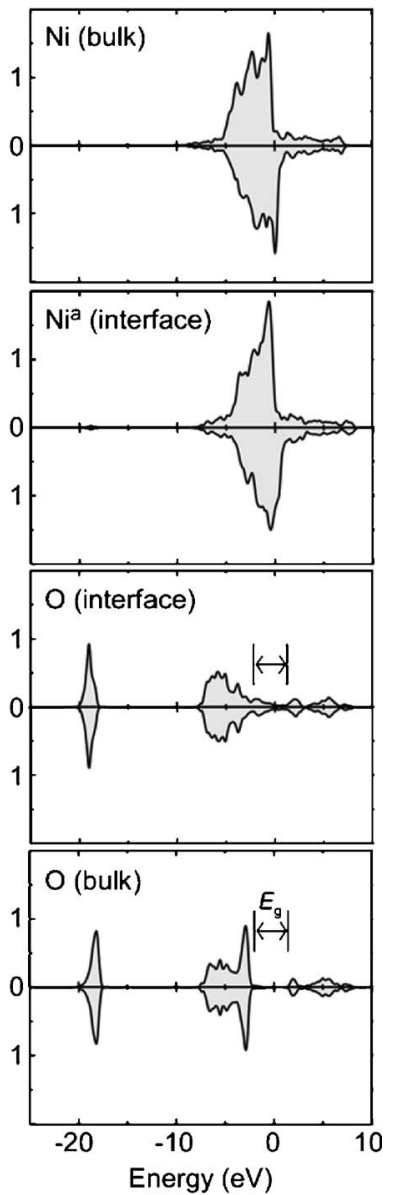

(b)
FIG. 4. Atom-projected DOS profiles for (a) the $\mathrm{Zr}$ terminated and (b) $\mathrm{O}$ terminated interface having the $T_{0}$-type structure. In these plots, DOSs for atoms around the centers of $\mathrm{Ni}$ and $\mathrm{ZrO}_{2}$ slabs (denoted as "bulk"), and those for interfacial $\mathrm{Ni}, \mathrm{Zr}$, and $\mathrm{O}$ in the on-top configuration are displayed. The Fermi level of each supercell is set at $0 \mathrm{eV}$.

degree of exchange splitting tends to decrease for $\mathrm{Ni}^{a}$ at the $\mathrm{Zr}$ terminated interface [Fig. 4(a)], and the calculated MM for $\mathrm{Ni}^{a}$ is found to be $0.19 \mu_{\mathrm{B}}$, which is quite smaller than that in bulk. This result is attributed with charge transfer from $\mathrm{ZrO}_{2}$ to $\mathrm{Ni}$. As stated before, the $\mathrm{Zr}$ terminated surface is $\mathrm{Zr}$ rich, and $\mathrm{Zr}$ is less electronegative than $\mathrm{Ni}$. Therefore, considerable charge transfer from $\mathrm{Zr}$ to $\mathrm{Ni}$ can take place in the interface formation. The transferred electrons from $\mathrm{ZrO}_{2}$ occupy the minority-spin orbital of Ni-3d originally unoccupied at the Fermi level, so that the Ni-3d orbitals are almost fulfilled by electrons and result in the similar DOS profiles between majority and minority spin, without exchange splitting. In contrast, the $\mathrm{Ni}^{a}$ PDOS at the $\mathrm{O}$ terminated interface does not change so significantly from that of bulk Ni, which is due to the fact that the $\mathrm{O}$ terminated $\mathrm{ZrO}_{2}$ surface is stoichiometric, and thus significant charge transfer does not necessarily occur in the interface formation. It is noted, however, that the $\mathrm{MM}$ of $\mathrm{Ni}^{a}$ in the $\mathrm{O}$ terminated case is calculated to be $0.50 \mu_{\mathrm{B}}$, and is slightly smaller than that of bulk $\mathrm{Ni}$.

PDOS curves for $\mathrm{Ni}$ at the different interfacial sites in the 


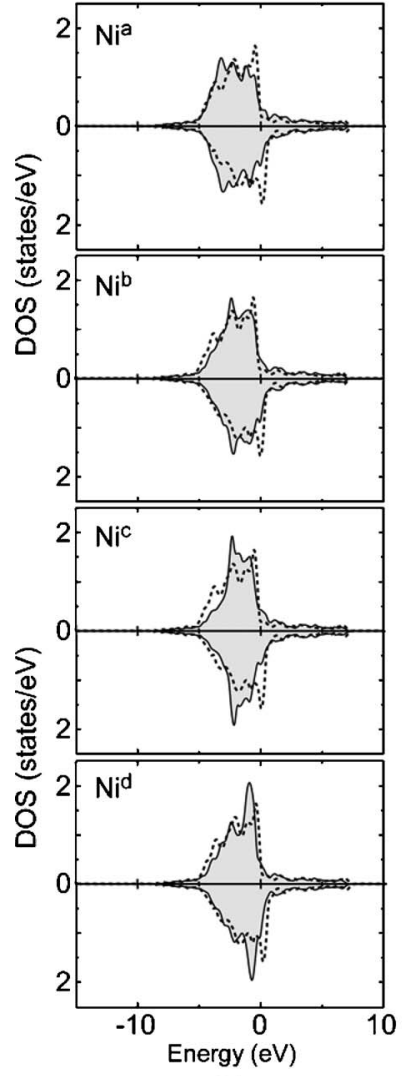

(a)

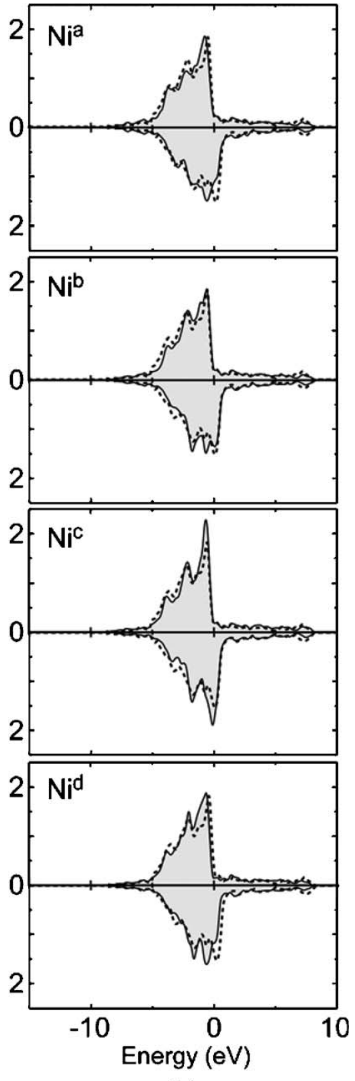

(b)
FIG. 5. DOS profiles for Ni atoms at different interfacial sites in (a) the $\mathrm{Zr}$ terminated and (b) the $\mathrm{O}$ terminated interface having the $T_{0}$-type structure. Dotted curves overlaid on each figure correspond to DOS profiles of Ni at the center of the Ni slab. The Fermi level of each supercell is set at $0 \mathrm{eV}$.

$\mathrm{Zr}$ and $\mathrm{O}$ terminated $T_{0}$-type structures are displayed in Fig. 5. In the case of $\mathrm{Zr}$ termination [Fig. 5(a)], it turns out that the PDOS profiles are varied depending on the Ni sites. The PDOS shape of $\mathrm{Ni}^{a}$ is quite different from that of bulk Ni, while the PDOS widths for other $\mathrm{Ni}$ atoms become narrower than those of $\mathrm{Ni}^{a}$ and bulk Ni. These features indicate different interactions of $\mathrm{Ni}-\mathrm{Zr}$ at the interface. In the $\mathrm{O}$ terminated case [Fig. 5(b)], differences of interfacial Ni PDOSs from that of bulk Ni are not so large as in the $\mathrm{Zr}$ terminated case, but it is apparently observed that each PDOS of minority spin at around the Fermi level is changed in a different way depending on Ni sites. Such slight PDOS changes indicate that orbital polarization or overlap between $\mathrm{Ni}$ and $\mathrm{O}$ may occur even at the $\mathrm{O}$ terminated interface. From the above results, it can be said that $\mathrm{Ni}$ atoms in the interface layer of each interface system are in site-dependent chemical environments with underlying $\mathrm{Zr}$ or $\mathrm{O}$ atoms.

In order to investigate site-dependent bonding characteristics at the interfaces in more detail, electron-density (ED) differences in the $T_{0}$-type interfaces are plotted in Fig. 6. Here, the EDs of the $\mathrm{Ni}$ and $\mathrm{ZrO}_{2}$ slabs having the same atomic positions with the interface supercells are subtracted from those of the interfaces. Thus the contour maps indicate bond-formation behavior between $\mathrm{Ni}$ and $\mathrm{ZrO}_{2}$ in the interface formation.
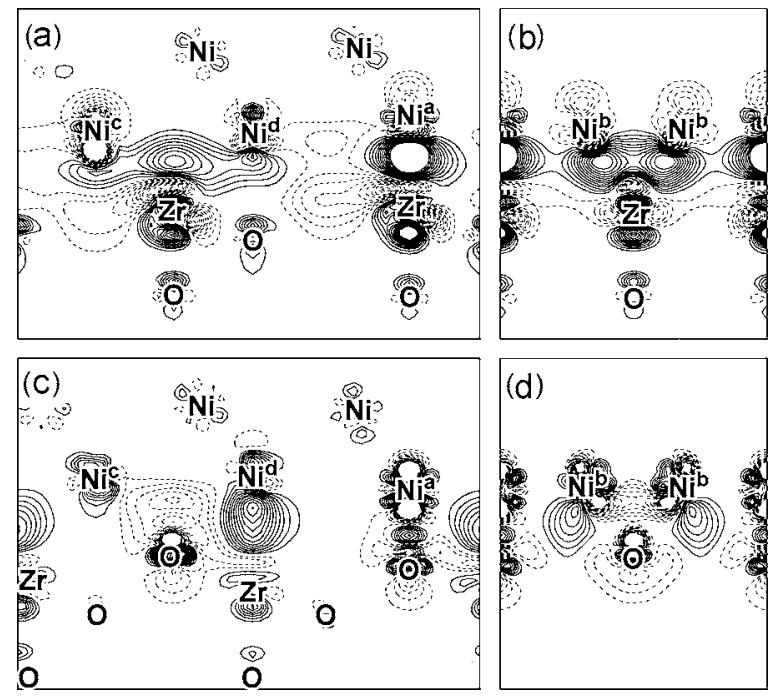

FIG. 6. ED difference maps for the $T_{0}$ structures of the $\mathrm{Zr}$ and $\mathrm{O}$ terminated interfaces. (a) and (c) are the plots on the (01) plane, while (b) and (d) are those on the $(\overline{2} 11)$ plane. The contour lines are drawn from -0.12 to 0.12 with an interval of 0.01 in electrons $/ \AA^{2}$, except for the zero contour line. Solid lines indicate positive values, while broken ones negative. On these cross sections, the on-top $\mathrm{Ni}$ $\left(\mathrm{Ni}^{a}\right)$, the two kinds of hollow-site $\mathrm{Ni}\left(\mathrm{Ni}^{c}\right.$ and $\left.\mathrm{Ni}^{d}\right)$ and the asymmetric-site $\mathrm{Ni}\left(\mathrm{Ni}^{b}\right)$ are present (also see Fig. 1).

In the $\mathrm{Zr}$ terminated case [Figs. 6(a) and 6(b)], it is obvious that the large areas with positive ED differences are present at the interface. This is due to that considerable charge transfer from $\mathrm{Zr}$ to $\mathrm{Ni}$ occurs and the electrons are sheared between atoms at the interface layers. Such features can also be understood from the DOS profiles shown in Fig. 5. As is seen in Fig. 6(a), the ED significantly increases between the on-top $\mathrm{Ni}$ atom $\left(\mathrm{Ni}^{a}\right)$ and $\mathrm{Zr}$, and the increased region is rather localized between the two atoms, which indicates their strong covalent interaction. The strong covalency of $\mathrm{Ni}^{a}-\mathrm{Zr}$ can also be understood from its bond length $(0.242 \mathrm{~nm})$, which is much smaller than the sum of their atomic radii $(0.285 \mathrm{~nm}) .{ }^{26}$ Around the hollow-site $\mathrm{Ni}^{c}$ and $\mathrm{Ni}^{d}$ atoms, the increase in ED is observed as well, but the electrons mainly concentrate above $\mathrm{Zr}$. Therefore, the positively charged $\mathrm{Zr}$ atom no longer has direct interactions with $\mathrm{Ni}^{c}$ and $\mathrm{Ni}^{d}$, but interacts with the electrons accumulated around the $\mathrm{Ni}$ interstitial region in an electrostatic (ionic) manner. Regarding the asymmetric-site $\mathrm{Ni}$ atoms $\left[\mathrm{Ni}^{b}\right.$ in Fig. 6(b)], the ED increases at around the midpoint with $\mathrm{Zr}$, and also the increased region is shared among the two neighboring $\mathrm{Ni}$ atoms. This indicates that the $\mathrm{Ni}-\mathrm{Zr}$ bonds can be described by covalent interactions with partial metallic characters. As shown above, the chemical interactions across the $\mathrm{Zr}$ terminated interface exhibit a site-dependent feature from ionic through covalent/metallic characters of bonding.

The increased ED regions at the $\mathrm{O}$ terminated interface [Figs. 6(c) and 6(d)] are much smaller than those in the $\mathrm{Zr}$ terminated case, since the $\mathrm{O}$ terminated $\mathrm{ZrO}_{2}$ surface is stoichiometric and thus large charge transfer does not necessarily take place. In such a case, ED redistribution in the $\mathrm{Ni}$ layers may occur due to the electrostatic field of the ionic 

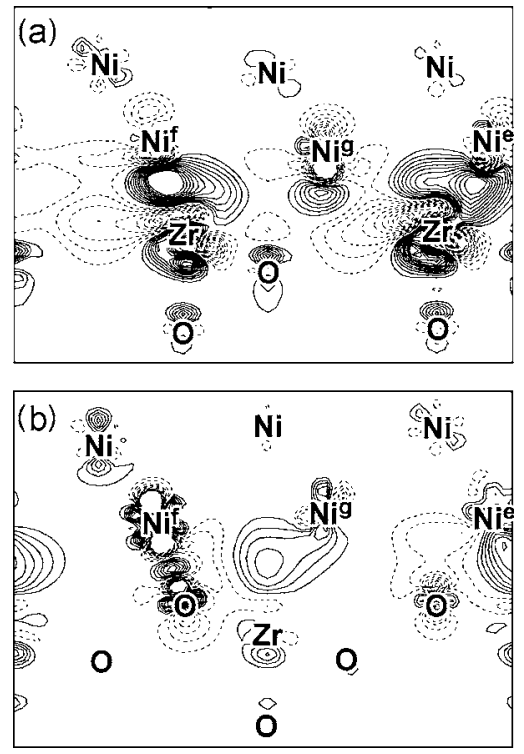

FIG. 7. ED difference maps on the $(01 \overline{1})$ planes for the $T_{1}$ structures of the $\mathrm{Zr}$ terminated (a) and $\mathrm{O}$ terminated (b) interfaces. The contour maps are drawn in the similar manner with Fig. 3.

$\mathrm{ZrO}_{2}$ layers, and the bonding of the $\mathrm{O}$ terminated interface may arise from image-chargelike interactions between $\mathrm{Ni}$ and $\mathrm{ZrO}_{2} \cdot{ }^{1,2}$ Obviously, the EDs of the $\mathrm{Ni}^{c}-\mathrm{Ni}^{d}$ interstitial regions decrease (positively charged), while the EDs just above $\mathrm{Zr}$ in the second $\mathrm{ZrO}_{2}$ layer increase (negatively charged). Such decrease and increase in ED can induce attractive ionic interactions with the oppositely charged $\mathrm{O}$ and $\mathrm{Zr}$ atoms, respectively. However, it is worth mentioning here that the on-top $\mathrm{Ni}^{a}$-O pair exhibits an increased ED region localized between the two atoms, indicating the covalent interaction. Therefore, charge rearrangement in the $\mathrm{Ni}$ layer does not always result in ionic interactions with $\mathrm{ZrO}_{2}$, but sometimes gives rise to interfacial covalent bonds depending on the bonding-pair configurations. This can be attributed to the fact that $\mathrm{Ni}-3 d$ orbitals originally have an electronunoccupied state, as stated in Figs. 4 and 5. The on-top $\mathrm{Ni}^{a}-\mathrm{O}$ configuration has a smaller bond length $(0.197 \mathrm{~nm})$ than the others (more than $0.21 \mathrm{~nm}$ ), and the Ni-3d orbitals atop $\mathrm{O}$ are subject to large polarization due to the negatively charged $\mathrm{O}$ atom [see Fig. 6(c)]. Although such detailed interactions of $\mathrm{Ni}^{a}-\mathrm{O}$ cannot easily be resolved only from the PDOS profiles shown in Fig. 5, the orbital hybridization of $\mathrm{Ni}-3 d$ with $\mathrm{O}-2 p$ occurs, which results in formation of the covalent $\mathrm{Ni}^{a}-\mathrm{O}$ bond. It can be said that the $\mathrm{O}$ terminated interface also exhibits a multiplicity of chemical bonds with site dependency, as a result of the ED redistribution and orbital polarization across the interface.

The feature of multiple bonding characters found for the $T_{0}$ structures is also applicable to the $T_{1}$ translation state. Figure 7 shows ED-difference maps for the $T_{1}$-type $\mathrm{Zr}$ and $\mathrm{O}$ terminated interfaces. In the $\mathrm{Zr}$ terminated case [Fig. 7(a)], the $\mathrm{Ni}$ atoms located at the sites slightly off from the exact on-top location $\left(\mathrm{Ni}^{e}\right.$ and $\left.\mathrm{Ni}^{f}\right)$ tend to form covalent bonds with $\mathrm{Zr}$. It is noted that the $\mathrm{Ni}^{f}-\mathrm{Zr}$ and $\mathrm{Ni}^{e}-\mathrm{Zr}$ bond lengths of $0.248 \mathrm{~nm}$ and $0.257 \mathrm{~nm}$ are close to the covalently bonded on-top $\mathrm{Ni}^{a}-\mathrm{Zr} \quad(0.242 \mathrm{~nm})$ and asymmetric $\mathrm{Ni}^{b}-\mathrm{Zr}$
$(0.256 \mathrm{~nm})$ lengths in the $T_{0}$ structure (see Fig. 6), respectively. Moreover, the electron-accumulated region manifests itself between $\mathrm{Ni}^{e}$ and $\mathrm{Ni}^{g}$, which contributes to ionic interactions with the $\mathrm{Zr}$ cation below. In the $\mathrm{O}$ terminated interface [Fig. 7(b)], the $\mathrm{Ni}^{f}-\mathrm{O}$ pair forms a covalent bond, and its bond length of $0.198 \mathrm{~nm}$ is quite similar to that of on-top $\mathrm{Ni}^{a}-\mathrm{O}(0.197 \mathrm{~nm})$ in the $T_{0}$ translation state [see Fig. 6(a)]. $\mathrm{Ni}-\mathrm{O}$ pairs with larger bond lengths $\left[\mathrm{Ni}^{e}-\mathrm{O}\right.$ and $\mathrm{Ni}^{g}-\mathrm{O}$ in Fig. 7(b)] no longer have covalent interactions. Alternatively, the increases in $\mathrm{ED}$ above $\mathrm{Zr}$ in the second layer, inducing their image-chargelike ionic interactions, can be observed. Even in the translated state, therefore, multiple chemical bonds ranging from ionic to covalent are realized in accordance with their local atomic configurations. It is noted, however, that the magnitudes of image charges induced at the interface are smaller than those in the $T_{0}$-type structure [see Fig. 6(c)]. The individual image charges induced are sensitive to the translation state.

It should be noted here that the similar $W_{\text {sep }}$ values in the two translation states for both the $\mathrm{Zr}$ and $\mathrm{O}$ terminations (shown in Fig. 3) should originate from the multiplicity of the interface bonding. If interface-energy differences among translation states are rather large, the energy penalty would be compensated by generation of misfit dislocations. For instance, the previous calculations of coherent-model $\mathrm{MgO} / \mathrm{Ag}(001)$ interfaces, which are in reality semicoherent with a lattice misfit of about $3 \%$, yielded a large energy difference of about $0.4 \mathrm{~J} / \mathrm{m}^{2}$ between different translation states. ${ }^{3}$ As compared to that, the changes in $W_{\text {sep }}$ due to the relative translations are much smaller in the present case (less than $0.04 \mathrm{~J} / \mathrm{m}^{2}$ ). Since the real incoherent interface structure can be represented by a mixture of the different translation states, the invariability of interface energy against the relative translation states may explain why the interface structures without long-range lattice continuity can be formed as a stable structure.

$W_{\text {sep }}$ values mean mechanical energy release upon interface fracture, and are used as a good measure of interface strength. In the case of coherent interfaces, $W_{\text {sep }}$ values correspond to the energy required to break interfacial bonds in a particular configuration. However, incoherent interfaces contain a multiplicity of interfacial bonds, and thus the situation upon interfacial fracture is more complicated, although the $W_{\text {sep }}$ values in Fig. 3 are independent of the translation states. In particular, energies to break chemical bonds at incoherent interfaces are also dependent on the individual bond characters, which should affect the mechanical response to applied stresses. In order to reveal an effect of the multiple interfacial bonding on mechanical properties of incoherent interfaces, first-principles tensile tests are performed for the incoherent $\mathrm{Ni} / \mathrm{ZrO}_{2}(111)$ interfaces.

In the present first-principles tensile tests, uniaxial tensile strains are imposed onto the stable interface structures. The supercell edge lengths normal to the interfaces (the [111] direction) are elongated in small increments, and all atomic positions are relaxed at each strained states. The supercell edge lengths parallel to the interfaces (the [101] and [ $\overline{1} 10]$ directions) are fixed throughout the tensile tests, preventing Poisson contraction, in order to reduce computational time. 

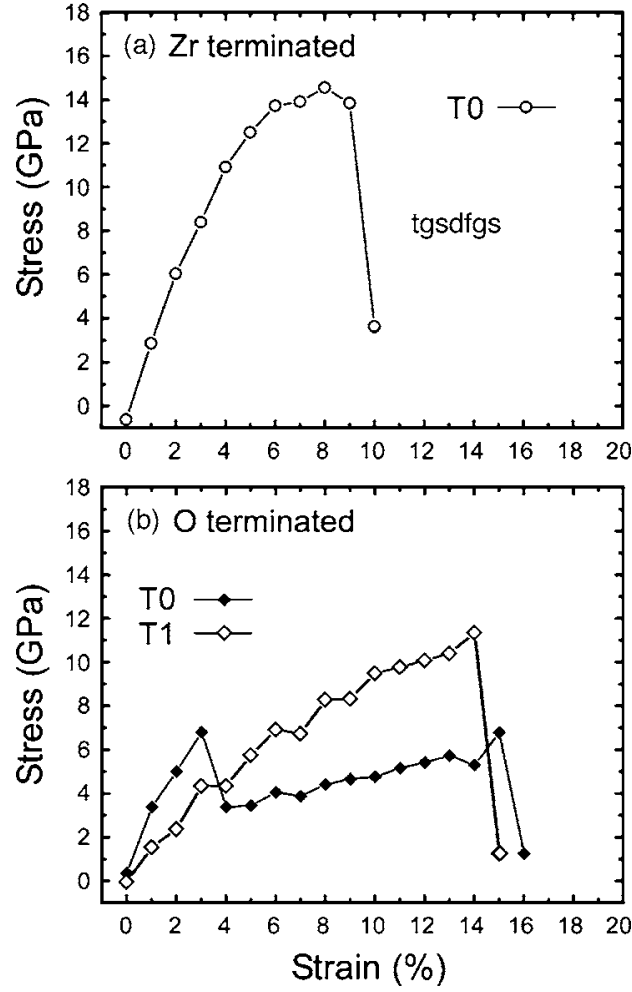

FIG. 8. The stress-strain curves for the $\mathrm{Zr}$ terminated and $\mathrm{O}$ terminated interfaces. The $T_{0}$ structure of the $\mathrm{Zr}$ terminated interface showed cleavage fracture inside $\mathrm{ZrO}_{2}$. In (a), the profile of $T_{0}$ is only displayed, but it is expected that the $T_{1}$-type structure shows the similar curve, since that translation state also have a rather large $W_{\text {sep }}$ value, resulting in cleavage inside $\mathrm{ZrO}_{2}$. In contrast, the $\mathrm{O}$ terminated interfaces exhibited fracture at the interface planes. However, it is interesting to point out the different stress-strain behaviors between the two translation states.

Strictly speaking, therefore, the present tensile tests correspond to uniaxial extension. Poisson contraction may decrease theoretical tensile stresses of bulk and grain boundaries, and thus maximum stresses for fracture without Poisson contraction are regarded as upper bounds of tensile strength of the incoherent interfaces. ${ }^{27,28}$

Figure 8 displays stress-strain curves of the $\mathrm{Ni} / \mathrm{ZrO}_{2}(111)$ interfaces. For the $T_{0}$-type $\mathrm{Zr}$ terminated interface [Fig. 8(a)], the stresses readily increase with rising strains, reach a maximum stress of $14.5 \mathrm{GPa}$ at the $8 \%$ strain, and abruptly drop at a strain of $10 \%$. In this case, the interface itself does not undergo fracture, and cleavage between $\mathrm{O}$ planes in the second and third $\mathrm{ZrO}_{2}$ layers from the interface plane actually takes place. It can be said that this stress-strain profile reflects typical brittle fracture behavior inside $\mathrm{ZrO}_{2}$. In fact, the $W_{\text {sep }}$ value for cleavage between (111) O planes in $\mathrm{ZrO}_{2}$ is found to be $1.69 \mathrm{~J} / \mathrm{m}^{2}\left(=2 \gamma_{\mathrm{s}}, \gamma_{\mathrm{s}}\right.$ is the surface energy), and is much smaller than those for cleavage between $\mathrm{Ni}(111)$ layers $\left(4.08 \mathrm{~J} / \mathrm{m}^{2}\right)$ and at the interface $\left(4.39 \mathrm{~J} / \mathrm{m}^{2}\right)$, which are obtained by calculations of separate $\mathrm{Ni}$ and $\mathrm{ZrO}_{2}$ surface slabs. In addition, it can be seen from interfacial bond-length changes against strains in Fig. 9(a) that the bond lengths across the interface do not exhibit notable changes against strains. Thus the interface bonding of the $\mathrm{Zr}$ terminated case

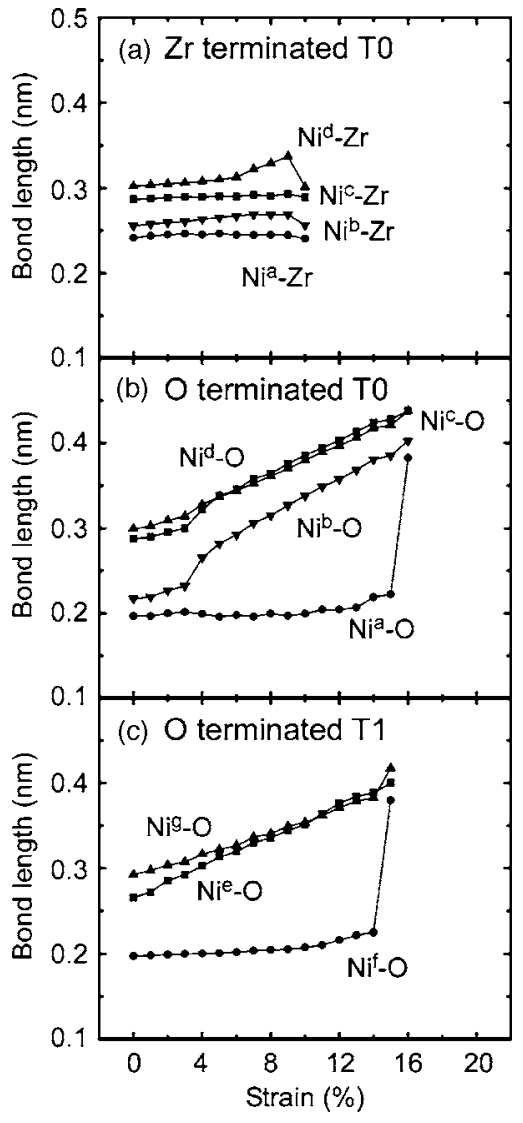

FIG. 9. The bond-length changes as a function of tensile strain. It is noted that the $T_{0}$-type interfaces contain four kinds of bondingpair configurations, whereas in the case of the $T_{1}$-type $\mathrm{O}$ terminated interface, three kinds of $\mathrm{Ni}-\mathrm{O}$ configurations with different bond lengths are present (also see Fig. 1).

is rather rigid against tensile loading. As shown in Fig. 3, the $\mathrm{Zr}$ terminated interface in the different translated state of $T_{1}$ exhibit the similar $W_{\text {sep }}$ value, and it can be expected that cleavage inside $\mathrm{ZrO}_{2}$ occurs, showing the quite similar stress-strain profile to Fig. 8(a).

In contrast, by the tensile tests of the $\mathrm{O}$ terminated interfaces, cleavage at the interface planes is observed, as expected from their small $W_{\text {sep }}$ values of around $0.5 \mathrm{~J} / \mathrm{m}^{2}$, whereas the stress-strain curves show different behavior from that of the $\mathrm{Zr}$ terminated case. In the case of the $T_{0}$ structure, the stresses increase almost linearly with strains up to $3 \%$, showing a maximum stress of $6.8 \mathrm{GPa}$, and yet decrease at the $4 \%$ strain. Subsequently, the stresses again gradually increase with increasing strains, and overall fracture throughout the interface plane eventually occurs at the $16 \%$ strain.

Such a stress-strain behavior of the $T_{0}$-type $\mathrm{O}$ terminated interface is attributed to different strength of individual chemical bonds across the interface. It can be seen from the bond-length changes [Fig. 9(b)] that Ni-O bonds in the hollow and asymmetric configurations $\left(\mathrm{Ni}^{b}-\mathrm{O}, \mathrm{Ni}^{c}-\mathrm{O}\right.$, and $\mathrm{Ni}^{d}{ }_{-}$ O) exhibit jumps in their bond-length profiles at the $4 \%$ strain, and thereafter their bond lengths readily increase with strains. This indicates that these Ni-O bonds start to break at a strain of $4 \%$, and no longer contribute to the interface 
adhesion for the further tensile strains. However, the $\mathrm{Ni}^{a}-\mathrm{O}$ bond maintains its length around $0.2 \mathrm{~nm}$ until the $15 \%$ strain, and the bond length rapidly increases at a strain of $16 \%$, meaning complete fracture of the interface. Therefore, the bonding strength of Ni-O in the on-top configuration, which has a covalent character shown in Fig. 6(c), is rather large as compared to those in the other configurations. Such a sitedependent bonding feature over the interface results in the distinct stress-strain profile of the $T_{0}$-type $\mathrm{O}$ terminated interface.

The $T_{1}$-type $\mathrm{O}$ terminated interface also exhibits a different stress-strain profile from the $T_{0}$-type one [Fig. 8(b)]. Considerable stress reduction during strain loading, as found in the $T_{0}$-type $\mathrm{O}$ terminated interface, is not observed in this case, but the profile for $T_{1}$ is not as smooth as that in the $\mathrm{Zr}$ terminated case. This is due to bond-breaking processes at the interfaces, beginning from the onset of strain loading. As can be seen in Fig. 9(c), the $\mathrm{Ni}^{e}-\mathrm{O}$ and $\mathrm{Ni}^{g}-\mathrm{O}$ pairs increase their distances monotonically with strains, which indicates that these Ni-O bonds are broken successively with strain. In contrast, the $\mathrm{Ni}^{f}-\mathrm{O}$ bond maintains its length of about $0.2 \mathrm{~nm}$ even for larger strains. In Fig. 7(b), the $\mathrm{Ni}^{f}-\mathrm{O}$ pair exhibits strong covalency, and thus the presence of the covalent bonds across the incoherent interface is responsible for the interface strength. In the $T_{1}$-type interface, three covalent $\mathrm{Ni}^{f}-\mathrm{O}$ pairs are present per unit [see Fig. 1(b)], which results in the larger maximum stress of about $11 \mathrm{GPa}$, as compared to that of the $T_{0}$-type interface $(6.8 \mathrm{GPa})$ with one covalent $\mathrm{Ni}^{a}-\mathrm{O}$ bond (on-top configuration) in the repeat unit. Owing to the on-top covalent pairs and their numbers contained in the translation states, the real incoherent $\mathrm{O}$ terminated interfaces do not undergo homogeneous cleavage over the interface plane. The fracture behavior is very sensitive to the translation states and their associated site-dependent interfacial bonding multiplicity.

In this paper, the first-principles results of the $\mathrm{Ni} / \mathrm{ZrO}_{2}(111)$ interfaces are reported, in order to reveal a bonding nature of incoherent metal/oxide interfaces. Even though the interface bonding is composed of one type of atomic-species pairs such as $\mathrm{Ni}-\mathrm{Zr}$ or Ni-O, depending on the interface termination, the interfacial atomic pairs exhibit sitedependent bonding characters. The bonding pair in the ontop configuration tends to have the strong covalent bond, while other Ni atoms in the hollow and other configurations form metallic or ionic for $\mathrm{Zr}$ termination, or ionic bonds for $\mathrm{O}$ termination. As compared to the covalent bond in the ontop configuration, such metallic or ionic interfacial interactions are neither directional nor rigid, which may stabilize the elongated or distorted bonding configurations intrinsically contained in the incoherent interfaces. Such a multiplicity of interfacial bonds also result in interface energetics independent of the translation states, but strongly affect the atomic-scale cleavage behavior of the incoherent interfaces. The bonding multiplicity cannot be understood by the image charge theory and previous coherent-model calculations, and must be new findings to depict general incoherent interfaces. This idea will thus be applicable to any heterointerface system as a generic bonding picture for large-mismatched interfaces.

This work was performed as a part of the Nanostructure Coating Project carried out by New Energy and Industrial Technology Development Organization. This work was also partly supported by a Grant-in-Aid for Scientific Research from the Ministry of Education, Sports, Science and Culture of Japan. K.M. also acknowledges useful advice by M. Kohyama at AIST, Japan, regarding computation of metal/oxide interfaces.
*Corresponding author. Electronic address: k.matsunaga @ materials.mbox.media.kyoto-u.ac.jp

${ }^{1}$ M. W. Finnis, J. Phys.: Condens. Matter 8, 5811 (1996).

${ }^{2}$ F. Ernst, Mater. Sci. Eng., R. 14, 97 (1995).

${ }^{3}$ T. Hong, J. R. Smith, and D. J. Srolovitz, Acta Metall. Mater. 43, 2721 (1995).

${ }^{4}$ G. L. Zhao, J. R. Smith, J. Raynolds, and D. J. Srolovitz, Interface Sci. 3, 289 (1996).

${ }^{5}$ I. G. Batirev, A. Alavi, M. W. Finnis, and T. Deutsch, Phys. Rev. Lett. 82, 1510 (1999).

${ }^{6}$ W. Zhang and J. R. Smith, Phys. Rev. B 61, 16883 (2000).

${ }^{7}$ A. Christensen and E. A. Carter, J. Chem. Phys. 114, 5816 (2001).

${ }^{8}$ J. I. Beltrán, S. Gallego, J. Cerdá, J. S. Moya, and M. C. Munoz, Phys. Rev. B 68, 075401 (2003).

${ }^{9}$ R. Benedek, A. Alavi, D. N. Seidman, L. H. Yang, D. A. Muller, and C. Woodward, Phys. Rev. Lett. 84, 3362 (2000).

${ }^{10}$ A. P. Sutton and R. W. Balluffi, Interfaces in Crystalline Solids (Oxford University Press, New York, 1996).

${ }^{11}$ Y. Ikuhara and P. Pirouz, Microsc. Res. Tech. 40, 206 (1998).

${ }^{12}$ G. Dehm, M. Rühle, G. Ding, and R. Raj, Philos. Mag. B 71, 1111 (1995).

${ }^{13}$ C. Scheu, W. Stein, and M. Rühle, Philos. Mag. A 78, 439 (1998).
${ }^{14}$ T. Sasaki, K. Matsunaga, H. Ohta, H. Hosono, T. Yamamoto, and Y. Ikuhara, Sci. Technol. Adv. Mater. 4, 575 (2003).

${ }^{15}$ T. Sasaki, K. Matsunaga, H. Ohta, H. Hosono, T. Yamamoto, and Y. Ikuhara, Mater. Trans., JIM 45, 2137 (2004).

${ }^{16}$ J. H. Van Der Merwe, J. Appl. Phys. 34, 117 (1963).

${ }^{17}$ Y. Ikuhara, P. Pirouz, A. H. Heuer, S. Yadavalli, and C. P. Flynn, Philos. Mag. A 70, 75 (1994).

${ }^{18}$ J. W. Matthews, Dislocations in Solids, edited by F. R. N. Nabarro, Vol. 2 (North-Holland Publishing, Amsterdam, 1979).

${ }^{19}$ G. Kresse and J. Furthmüller, Comput. Mater. Sci. 6, 15 (1996).

${ }^{20}$ P. E. Blöchl, Phys. Rev. B 50, 17953 (1994).

${ }^{21}$ G. Kresse and D. Joubert, Phys. Rev. B 59, 1758 (1999).

${ }^{22}$ J. P. Perdew, J. A. Chevary, S. H. Vosko, K. A. Jackson, M. R. Pederson, D. J. Singh, and C. Fiolhais, Phys. Rev. B 46, 6671 (1992).

${ }^{23}$ H. J. Monkhorst and J. D. Pack, Phys. Rev. B 13, 5188 (1976).

${ }^{24}$ J. H. Cho and M. H. Kang, Phys. Rev. B 52, 9159 (1995).

${ }^{25}$ E. G. Moroni, G. Kresse, J. Hafner, and J. Furthmüller, Phys. Rev. B 56, 15629 (1997).

${ }^{26}$ C. Kittel, Introduction to Solid State Physics, 6th ed. (John Wiley \& Son, Inc., New York, 1986).

${ }^{27}$ M. Kohyama, Philos. Mag. Lett. 79, 659 (1999).

${ }^{28}$ G. H. Lu, S. Deng, T. Wang, M. Kohyama, and R. Yamamoto, Phys. Rev. B 69, 134106 (2004). 